\title{
Low-dose hydrocortisone replacement therapy is associated with improved bone remodelling balance in hypopituitary male patients
}

\section{Lucy-Ann Behan', Grainne Kelleher², Mark J Hannon', Jennifer J Brady ${ }^{3}$, Bairbre Rogers ${ }^{1}$, William Tormey ${ }^{2}$, D Smith ${ }^{1}$, Christopher J Thompson ${ }^{1}$, Malachi J McKenna ${ }^{3,4}$ and Amar Agha ${ }^{1}$}

Departments of ${ }^{1}$ Endocrinology, Diabetes and Metabolism and ${ }^{2}$ Chemical Pathology, Beaumont Hospital, Dublin, Ireland, ${ }^{3}$ Metabolism Laboratory, St Vincent's University Hospital, Dublin, Ireland and ${ }^{4}$ School of Medicine and Medical Science, University College Dublin, Dublin, Ireland
Correspondence should be addressed to A Agha

Email

amaragha@beaumont.ie

\begin{abstract}
Objective: Glucocorticoid (GC) therapy is associated with adverse effects on bone metabolism, yet the effects of different GC physiological replacement regimens in hypopituitarism are not well characterised. We aimed to assess the effect of three hydrocortisone $(\mathrm{HC})$ replacement dose regimens on bone turnover.

Study design: An open cross-over study randomising ten hypopituitary men with severe ACTH deficiency to three commonly used $\mathrm{HC}$ dose regimens: dose $\mathrm{A}$ ( $20 \mathrm{mg}$ mane and $10 \mathrm{mg}$ tarde), dose $B$ ( $10 \mathrm{mg}$ mane and $10 \mathrm{mg}$ tarde) and dose $\mathrm{C}$ (10 $\mathrm{mg}$ mane and $5 \mathrm{mg}$ tarde).

Methods: Following 6 weeks of each regimen, the participants underwent 24-h serum cortisol sampling and measurement of bone turnover markers: bone-specific alkaline phosphatase, procollagen type I N-propeptide (PINP), intact osteocalcin (OC(1-49)),

C-terminal cross-linking telopeptide (CTX-I) and tartrate-resistant acid phosphatase $5 \mathrm{~b}$ (TRACP5b). Bone remodelling balance was estimated as an absolute ratio (PINP:CTX-I) and as an index using standardised scores derived from the matched controls.

Results: There were significant increases in the concentrations of the formation markers PINP $(P=0.045)$ and OC(1-49) $(P=0.006)$ and in the PINP:CTX-I ratio $(P=0.015)$, and a more positive bone remodelling balance index $(P=0.03)$ was observed in patients on the lowest dose $C$ than in those on the highest dose A. Mean 24-h cortisol concentrations correlated negatively with CTX-I ( $r=-0.66$ and $P=0.04)$ and TRACP5b $(r=-0.74$ and $P=0.01)$ in patients on dose $B$ and with OC(1-49) $(r=-0.66$ and $P=0.04)$ and CTX-I $(r=-0.81$ and $P<0.01)$ in patients on dose $C$. In patients receiving the lower-dose regimen, trough cortisol concentrations correlated with increased bone formation and resorption.

Conclusion: Low-dose $\mathrm{HC}$ replacement (10 $\mathrm{mg}$ mane and $5 \mathrm{mg}$ tarde) is associated with increased bone formation and a positive bone remodelling balance. This may have a long-term beneficial effect on bone health.
\end{abstract}

\section{Introduction}

The optimisation of glucocorticoid (GC) replacement in adrenocorticotrophin (ACTH)-deficient hypopituitary subjects in clinical practice remains challenging and GC excess has long been reported to be associated with osteoporosis $(1,2)$. The adverse effects of GCs on bone health have largely been demonstrated in studies using supraphysiological doses of GC for anti-inflammatory therapeutic effects $(3,4,5)$; however, an increased relative (c) 2014 European Society of Endocrinology Printed in Great Britain
Published by Bioscientifica Ltd. 
risk of 1.55 (95\% CI 1.2-2.01) for vertebral fractures has also been observed with the long-term use of doses of prednisolone $<2.5 \mathrm{mg} /$ day (6).

Although GC replacement therapy for primary and secondary adrenal insufficiency aims to use physiological dose regimens, there is conflicting evidence that GC replacement is also associated with deleterious effects on bone. The majority of available studies have been carried out in subjects with primary adrenal failure, with some studies finding a reduced bone mineral density (BMD) in men with increasing hydrocortisone (HC) equivalent doses $(7,8)$ and a lower femoral neck BMD correlated with weight-adjusted GC dose (9), while others have found no difference in BMD compared with that of the control population $(10,11)$, except in subjects on prednisolone, in whom a significant decrease in BMD was observed (12).

There is a paucity of data on the effect of GC replacement on bone metabolism in patients with ACTH deficiency. Peacey et al. (13) demonstrated in a cross-sectional and prospective cohort of 32 patients, 20 of whom had secondary adrenal insufficiency, that a reduction in GC dose by $30 \%$, to $20 \mathrm{mg}$ daily, was associated with a 19\% increase in the concentrations of intact osteocalcin (OC(1-49)), a marker of bone formation, and a weak but significant negative correlation between absolute BMD and HC dose used in replacement. This finding was replicated in an observational study carried out by Chikada et al. (14) in a group of primary $(n=10)$ and secondary $(n=5)$ hypoadrenal patients in whom a negative correlation between $\mathrm{HC}$ dose and BMD and also cumulative $\mathrm{HC}$ dose and BMD was observed. Wichers et al. (15) prospectively randomised nine patients to three different HC dose regimens in a double-blind study and also demonstrated a significant increase in the concentrations of $\mathrm{OC}(1-49)$ as the dose of HC decreased from 30 to $15 \mathrm{mg}$; however, there was no control group as well as no comment on the replacement status of the other pituitary hormones, which can have significant effects on bone turnover.

The aim of the present study was to determine in a prospective, cross-over randomised controlled manner the effect of three commonly used $\mathrm{HC}$ replacement regimens on bone turnover markers in a group of male hypopituitary patients, fully replaced on all other pituitary hormones, including growth hormone (GH).

\section{Subjects and methods}

\section{Patients and controls}

Ten adult hypopituitary men with known severe ACTH deficiency, defined by a fasting morning total serum cortisol concentration $<100 \mathrm{nmol} / \mathrm{l}$ and a stimulated peak cortisol concentration $<400 \mathrm{nmol} / \mathrm{l}$ in response to insulin-induced hypoglycaemia, were included in this study.

All ten subjects had been diagnosed and treated for sellar tumours between 3 and 18 years prior to the inclusion in the study. Five patients had been treated for non-functioning pituitary adenoma, two for macroprolactinoma, two for craniopharyngioma and the 10th had treated, cured, Cushing's disease with panhypopituitarism and was 8 years post definitive treatment, still requiring $\mathrm{HC}$ replacement, with a morning pre-HC cortisol level $<100 \mathrm{nmol} / \mathrm{l}$. Nine patients had complete anterior pituitary failure, and one patient was deficient in all the anterior pituitary hormones, except luteinising hormone and follicle-stimulating hormone; all the patients were on appropriate replacement of hormones including GH, without alteration in dose for at least 3 months prior to and during the study. All the ten patients had diabetes insipidus and were on desmopressin. No patient had serum sodium abnormalities to suggest under- or over-replacement with desmopressin. Anterior pituitary hormone replacement therapy regimens were not altered during the study period, except for HC dose as per study protocol. No patient was on calcium or vitamin D supplementation.

Exclusion criteria were age $<18$ years, advanced medical or surgical illness, conditions associated with altered bone turnover such as Paget's disease of bone, known osteoporosis or fracture within the previous 1 year. We excluded patients on GCs for reasons other than ACTH deficiency and those on agents that interfere with corticosteroid metabolism or bone metabolism such as antiepileptic medications (16). Female patients were excluded because of the variable effects of oestrogen status on bone turnover marker measurement (17) and also on corticosteroid-binding globulin (CBG) concentrations, thus affecting total cortisol concentrations and cortisol kinetics (18).

Healthy male controls $(n=10)$, matched for age, BMI and waist circumference (WCM), were enrolled to undergo the same biochemical investigations and clinical examination as the patient group.

All the patients and controls gave written informed consent to participate in this study, which was approved by the Beaumont Hospital Medical Research (Ethics) Committee and the Irish Medicines Board.

\section{Study design}

In this prospective clinical trial, all ten patients were randomised in an open cross-over protocol to take three commonly prescribed doses of HC: dose A (20 mg at 
$0800 \mathrm{~h}$ and $10 \mathrm{mg}$ at $1600 \mathrm{~h})$, dose B (10 $\mathrm{mg}$ at 0800 and $1600 \mathrm{~h}$ ) or dose C (10 $\mathrm{mg}$ at $0800 \mathrm{~h}$ and $5 \mathrm{mg}$ at $1600 \mathrm{~h})$. In view of the short half-life of $\mathrm{HC}$, the patients followed each dose regimen for a total of 6 weeks to allow adequate time for a 'washout' of the previous dose. At the end of each 6-week treatment schedule, the patients were admitted to our clinical research centre overnight. Following a physical examination, blood samples were drawn through a heparinised i.v. cannula hourly, for $24 \mathrm{~h}$, for the measurement of cortisol concentrations. Basal free thyroxine, thyrotrophin, testosterone, sex hormonebinding globulin, albumin, gonadotrophins, prolactin and insulin-like growth factor 1 (IGF1), parathyroid hormone (PTH), 25-hydroxyvitamin D (25OHD), calcium and albumin concentrations and renal function were measured upon the commencement of each 24-h admission. Details regarding the effects of these dose regimens on serum cortisol profiles and quality of life of this cohort have been published previously, and this protocol has been described in detail in the work of Behan et al. (19). To control for circadian variation and food intake effect on bone turnover markers $(20,21)$, the subjects fasted from midnight during the admission and the morning dose of HC was withheld until the completion of venous sampling for bone turnover markers between 0730 and $0800 \mathrm{~h}$ (see below). Samples were centrifuged at 3000 r.p.m. for $15 \mathrm{~min}$ and stored in $1 \mathrm{ml}$ aliquots at $-80^{\circ} \mathrm{C}$ until analysis. Ten healthy matched controls underwent the same biochemical profiling as the patient group.

\section{Laboratory methods}

Bone formation markers $>$ The concentrations of OC (1-49) were measured using an electrochemiluminescence immunoassay on the Elecsys 2010 analyser (Roche Diagnostics) with intra-assay coefficients of variation (CV) of $4.0,3.3$ and $1.4 \%$ and inter-assay $\mathrm{CV}$ values of $6.5,3.8$ and $1.8 \%$ at concentration values of $15.5,13.7$ and $68.3 \mu \mathrm{g} / \mathrm{l}$ respectively and a lower limit of detection of $0.500 \mu \mathrm{g} / \mathrm{l}$. Normal reference ranges are age and sex dependent. The concentrations of procollagen type I N-propeptide (PINP) were measured on the same analyser (Roche Diagnostics) with intra-assay CV values of 1.8, 2.1 and $2.9 \%$ and inter-assay $\mathrm{CV}$ values of $2.3,2.4$ and $3.7 \%$ at concentration values of 274,271 and $799 \mu \mathrm{g} / 1$ respectively and a lower limit of detection of $5 \mu \mathrm{g} / \mathrm{l}$. The concentrations of bone-specific alkaline phosphatase (bone ALP), a marker of both bone mineralisation and maturation, were measured by an immunoenzymatic assay (Immunodiagnostic Systems Ltd, Bolton, UK) on an automated
ELISA platform with inter-assay and intra-assay CV values of $5.8 \%$ (at $8.4 \mu \mathrm{g} / \mathrm{l}$ ) and $6.5 \%$ (at $7 \mu \mathrm{g} / \mathrm{l}$ ) respectively.

Bone resorption markers - The concentrations of C-terminal cross-linking telopeptide (CTX-I) were measured using an electrochemiluminescence immunoassay on the Elecsys 2010 analyser (Roche Diagnostics) with intra-assay CV values of 4.6, 1.8 and $1.0 \%$ and inter-assay CV values of $4.7,4.3$ and $1.6 \%$ at concentration values of $0.08,0.39$ and $3.59 \mu \mathrm{g} / 1$ respectively. The lower detection limit was $0.01 \mu \mathrm{g} / \mathrm{l}$. The concentrations of tartrateresistant acid phosphatase 5b (TRACP5b) were measured by ELISA (Immunodiagnostic Systems Ltd) with interassay and intra-assay CV values of $4.25 \%$ (at 3.20 IU/l) and $4.7 \%$ (at $5 \mathrm{IU} / 1)$ respectively.

Bone remodelling indices $\downarrow$ As the normal reference range of bone turnover markers is dependent on age and sex, we calculated each result in s.D. units, the $Z$-score, using data obtained from the healthy age-, sex-, BMI- and WCM-matched control group. The $Z$-score was calculated as follows: $Z=(x-\mu) / \mathrm{o}^{\prime}$, where $x$ is the individual bone marker value, $\mu$ is the mean bone marker value of controls of the same gender and age, and $\mathrm{o}^{\prime}$ is the s.D. of bone marker value of controls of the same gender and age. Using the $Z$-scores, we were then able to calculate the bone remodelling balance index as follows: (formation (PINP) $Z$-score - resorption (CTX-I) Z-score) $(22,23)$. We also calculated the PINP:CTX-I ratio as an approximation of bone remodelling balance $(24,25)$.

Other biochemical indices $\downarrow$ The serum concentrations of $25 \mathrm{OHD}$ were measured by a competitive RIA (Immunodiagnostic Systems Ltd) as described previously (26). The serum concentrations of PTH were measured using an electrochemiluminescence immunoassay on the Elecsys 2010 analyser (Roche Diagnostics) with intra-assay CV values of $2.7,1.6$ and $1.5 \%$ and inter-assay $C V$ values of $6.5,3.9$ and $3.0 \%$ at concentration values of $26.7,52.5$ and $261 \mathrm{ng} / 1$ respectively. The normal reference range is 15-65 ng/l (1.6-6.9 pmol/l) based on the manufacturer's guidelines. Renal function and albumin and calcium concentrations were measured using the Beckman Coulter AU5400 by standard laboratory protocols. Serum cortisol, serum IGF1, thyroid function, testosterone, prolactin and CBG measurements have been described elsewhere (19). Anterior and posterior pituitary function was assessed using a standard methodology as described previously (19). Peak morning and afternoon cortisol levels were based on a single maximum cortisol level post $\mathrm{HC}$ 
Table 1 Data of patients and controls. Results expressed as means \pm s.D.

\begin{tabular}{|c|c|c|c|}
\hline & $\begin{array}{c}\text { Patients } \\
(n=10)\end{array}$ & $\begin{array}{c}\text { Controls } \\
(n=10)\end{array}$ & $P$ value \\
\hline Age (years) & $46 \pm 15$ & $45 \pm 15$ & 0.9 \\
\hline BMI $\left(\mathrm{kg} / \mathrm{m}^{2}\right)$ & $29.8 \pm 5.3$ & $29.1 \pm 4.6$ & 0.5 \\
\hline WCM $(\mathrm{cm})$ & $105 \pm 14$ & $103 \pm 11$ & 0.53 \\
\hline Basal cortisol (nmol/l) & $76.8 \pm 6.5$ & $403.3 \pm 122.4$ & $<0.001 *$ \\
\hline Free $\mathrm{T}_{4}(\mathrm{pmol} / \mathrm{l})$ & $11.3 \pm 2.1$ & $10.9+1.0$ & 0.6 \\
\hline IGF1 $(\mu \mathrm{g} / \mathrm{l})$ & $163 \pm 45$ & $152+32$ & 0.5 \\
\hline Testosterone (pmol/l) & $14.2 \pm 4.1$ & $16.4 \pm 7.7$ & 0.4 \\
\hline Creatinine $(\mu \mathrm{mol} / \mathrm{l})$ & $100 \pm 5$ & $77 \pm 3$ & $0.001 *$ \\
\hline GFR $\left(\mathrm{ml} / \mathrm{min}\right.$ per $\left.1.73 \mathrm{~m}^{2}\right)$ & $73 \pm 5$ & $97.6 \pm 5$ & $0.004 *$ \\
\hline Serum calcium (mmol/l) & $2.32 \pm 0.01$ & $2.30 \pm 0.02$ & 0.47 \\
\hline Albumin $(g / l)$ & $40 \pm 0.8$ & $39 \pm 2$ & 0.66 \\
\hline PTH (ng/l) & $41 \pm 3$ & $32 \pm 2$ & 0.06 \\
\hline $250 \mathrm{OHD}(\mathrm{nmol} / \mathrm{l})$ & $49 \pm 3.9$ & $56 \pm 3$ & 0.17 \\
\hline
\end{tabular}

WCM, waist circumference.

administration for each patient. 'Day-time' trough levels were based on a minimum single level prior to the next dose at $1600 \mathrm{~h}$ afternoon, while 'night-time' trough levels were based on the single minimum cortisol level after the afternoon dose, but before $0200 \mathrm{~h}$ in order to account for the lack of physiological morning cortisol rise.

\section{Statistical analysis}

Results are reported as means (s.D.) or medians (interquartile ranges, IQRs) as appropriate. Bone turnover markers and other biochemical indices were analysed for normality using the D'Agostino-Pearson normality test. Between-group differences were assessed using ANOVA or repeated-measures ANOVA or the non-parametric equivalent, followed by the application of a multiple comparison test. Correlations were analysed using the Spearman or Pearson correlation coefficient, as appropriate based on normality tests. Significance was defined for $P$ values $<0.05$.

\section{Results}

The baseline characteristics of the patients and controls are given in Table 1 . The patients were age, sex, BMI and WCM matched with controls. There were no differences in the levels of thyroid hormone, testosterone, IGF1, 25OHD or PTH measured between patients on stable pituitary replacement therapy and healthy controls (Table 1). Although the control group had better renal function than the patients, based on the estimated glomerular filtration rate (eGFR) and serum creatinine concentrations (Table 1), only one patient had an eGFR $<60 \mathrm{ml} / \mathrm{min}$ per $1.73 \mathrm{~m}^{2}$ and there was no difference in renal function between patients on the three dose regimens $(P=0.8$; Table 2). Serum electrolyte levels were recorded at each visit, and there were no abnormalities suggestive of overor under-replacement with ddAVP and nor was there any difference in 24-h urine volume between patients under the three dose regimens or between the patients and controls ( $P=0.18$ and $P=0.7$ respectively). There was no correlation between 25OHD and PTH concentrations when the whole group was analysed $(r=-0.04$ and $P=0.8)$ or when only patients $(r=0.02$ and $P=0.9)$ or only controls $(r=0.08$ and $P=0.8)$ were analysed.

Table 2 Comparison of renal and bone indices between dose regimens and with controls. Data are expressed as means \pm s.D. or medians (interquartile ranges).

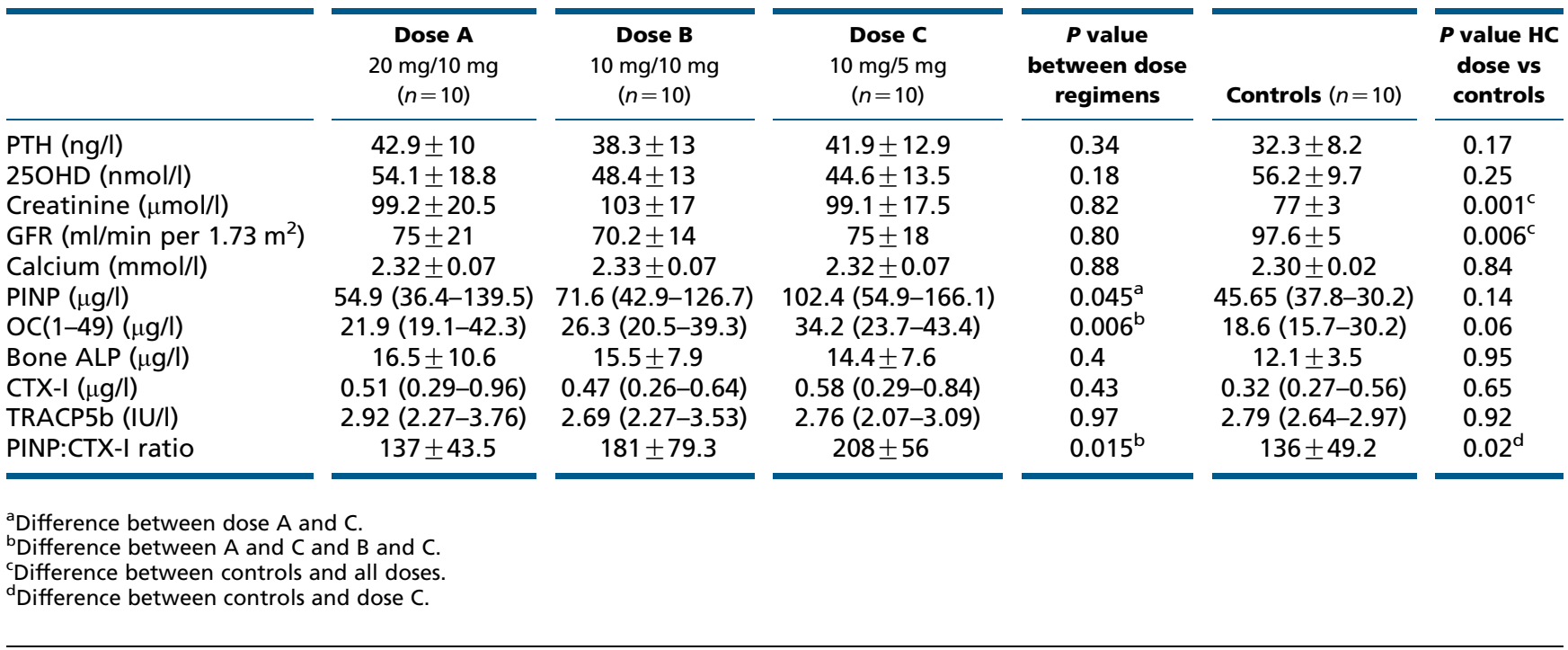


There was also no correlation between renal function or 25OHD ( $r=0.2$ and $P=0.2)$ or PTH $(r=-0.15$ and $P=0.4)$ concentrations when the whole group was analysed or when the patients and controls were analysed separately. No patient or control had PTH concentrations above the normal reference range of $65 \mathrm{ng} / \mathrm{l}$.

\section{Bone turnover markers}

The concentrations of bone formation markers, PINP and OC(1-49), were significantly higher in patients on doses B and $\mathrm{C}$ than in those on dose A. The concentrations of PINP were $86 \%$ higher and those of OC were $56 \%$ higher in patients on dose $\mathrm{C}$ than in those on dose $\mathrm{A}$ (Table 2). There was no difference in the concentrations of bone ALP or in those of the two markers of bone resorption, CTX-I and TRACP5b (Table 2).

There was no correlation between the mean 24-h cortisol concentrations and any bone turnover marker in patients on dose A or in controls. However, mean 24-h cortisol concentrations in patients on dose B demonstrated a moderate, non-significant negative correlation with OC(1-49), PINP and bone ALP and a strong, significant negative correlation with CTX-I and TRACP5b (Fig. 1). In patients on the lowest-dose regimen, dose C, there was a moderate-to-strong, negative correlation
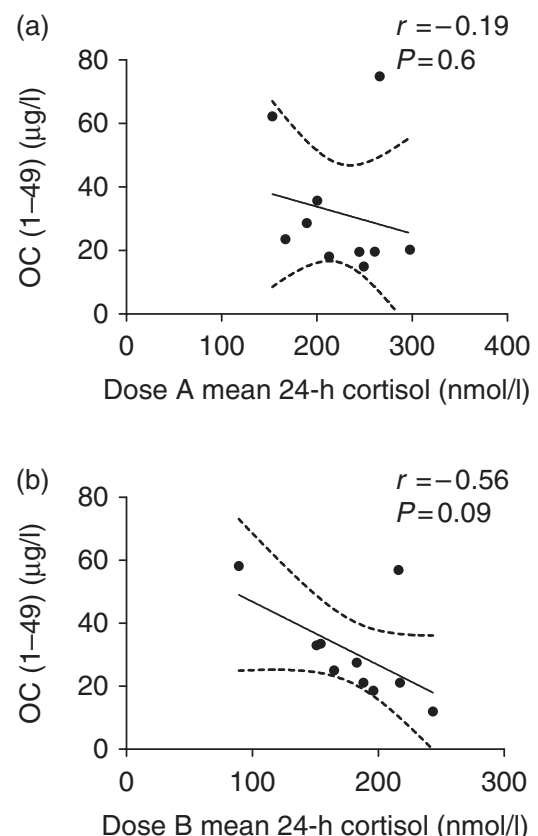

Dose B mean 24-h cortisol (nmol/l)
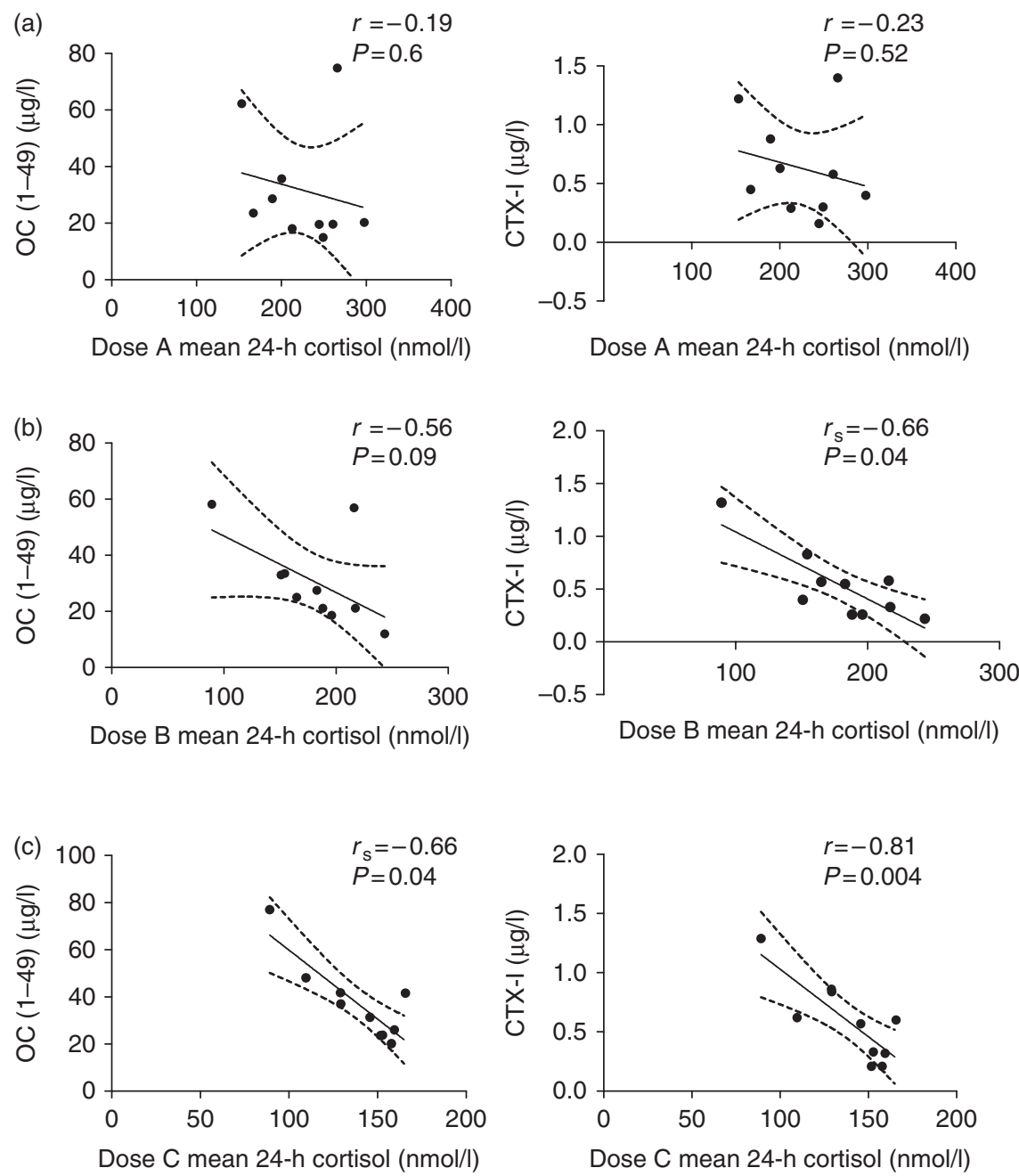
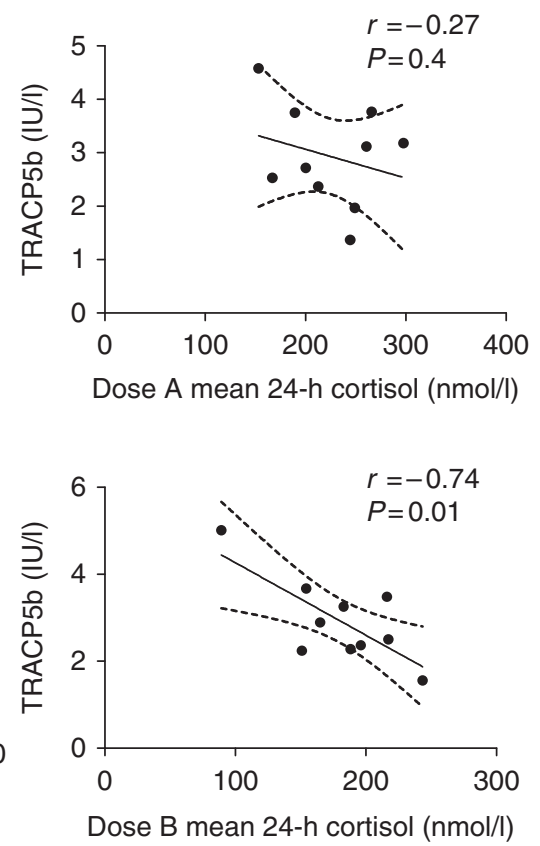

Dose B mean 24-h cortisol (nmol/l)

\section{Figure 1}

Correlations between mean 24-h cortisol concentrations and selected bone turnover markers for dose regimens. $r$, Pearson's correlation; $r_{\mathrm{s}}$, Spearman's correlation. (a) Dose $\mathrm{A}$, (b) dose B, and (c) dose C. 
between mean 24-h cortisol concentrations and each bone turnover marker; however, only correlations for OC(1-49) and CTX-I were statistically significant (Fig. 1). When data on the mean 24-h cortisol concentrations of patients regardless of the dose regimen followed were pooled, we found a moderate, significant negative correlation for OC(1-49) and PINP and a nearly significant, negative correlation for CTX-I (Supplementary Figure 1, see section on supplementary data given at the end of this article).

There was no correlation between peak serum cortisol concentrations after the administration of either the morning or afternoon dose of $\mathrm{HC}$ and any bone turnover marker in patients under the three dose regimens (Supplementary Figure 2). In patients on dose C, daytime trough levels demonstrated strong, significant negative correlations with all the bone turnover markers, as shown in Fig. 2, while there were moderate, negative correlations between the night-time trough levels and bone turnover markers, which were non-significant, except for OC(1-49) $(r=-0.64$ and $P=0.05)$ (Supplementary Figure 2).
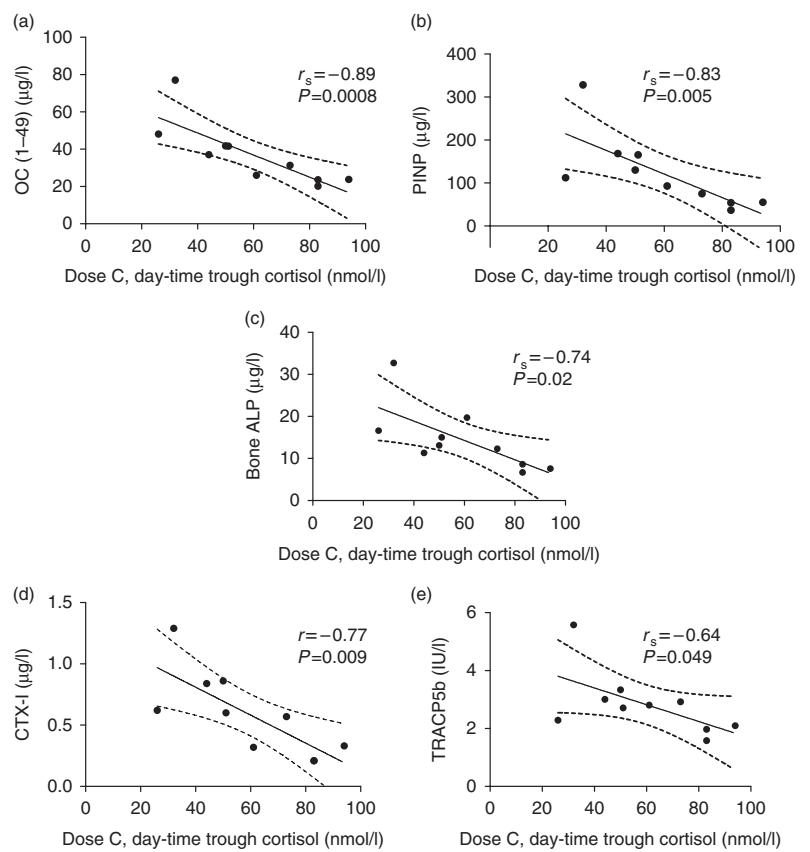

\section{Figure 2}

Trough cortisol concentrations following the administration of the morning $\mathrm{HC}$ dose and their correlation with bone turnover markers. ( $a, b$, and $c$ ) Bone formation and ( $d$ and e) bone resorption. $r$, Pearson's correlation; $r_{\mathrm{s}}$, Spearman's correlation.

\section{Bone remodelling balance and bone marker indices}

There was a significant increase in the formation $Z$-score to a median (IQR) of $+1.81(0.03-4.19)$ when the patients were on dose $\mathrm{C}$ (Table 2), whereas there was no significant difference in the resorption $Z$-scores between patients on the three dose regimens. The bone remodelling balance index in patients on dose $\mathrm{C}$ exhibited a significantly positive remodelling balance compared with that in patients under the other dose regimens $(P=0.03)$ (Fig. 3a). The PINP:CTX-I ratio was significantly higher in patients on dose $C(208.7 \pm 56.5)$ than in those under the other dose regimens (A: $137.5 \pm 43.5$; B: $181.7 \pm 79.3$ ) $(P=0.015)$ (Fig. 3b), consistent with increased bone formation under this dose. The ratio was also higher in patients on dose $C$ than in controls $(136.3 \pm 49.2)$ $(P=0.02)$.

There was no correlation between renal function or serum PTH concentrations and any of the bone turnover markers when analysed for the whole group, only patients or only controls (data not shown). However, there was a negative correlation between 25OHD and PINP $(r=-0.39$ and $P=0.01), O C(1-49)(r=-0.39$ and $P=0.01)$, CTX-I $(r=-0.32$ and $P=0.04)$ and bone ALP $(r=-0.43$ and $P=0.006)$ concentrations, but not for TRACP5b concentrations ( $r=-0.24$ and $P=0.12$ ), in the whole group. This correlation was lost when the patients and controls were analysed separately.

\section{Discussion}

In this prospective, randomised controlled cross-over study on the effects of three commonly used HC replacement regimens in panhypopituitary subjects on full pituitary replacement, we demonstrated that using a lower dose of HC leads to increased concentrations of bone formation markers, without a significant change in those of resorption markers. We also demonstrated a relationship between 24 -h serum cortisol profile and bone formation markers, particularly between trough serum cortisol concentrations and both bone formation and resorption markers. Our results indicate that lower cortisol exposure in hypopituitary patients, receiving what would be regarded as 'physiological' HC replacement, leads to a positive bone remodelling balance with increased bone formation, both of which are associated with a more favourable bone effect, and that cortisol dynamics may be as important to bone turnover as the overall dose exposure. These results are significant, particularly since it is clear that in clinical practice hypopituitary subjects 

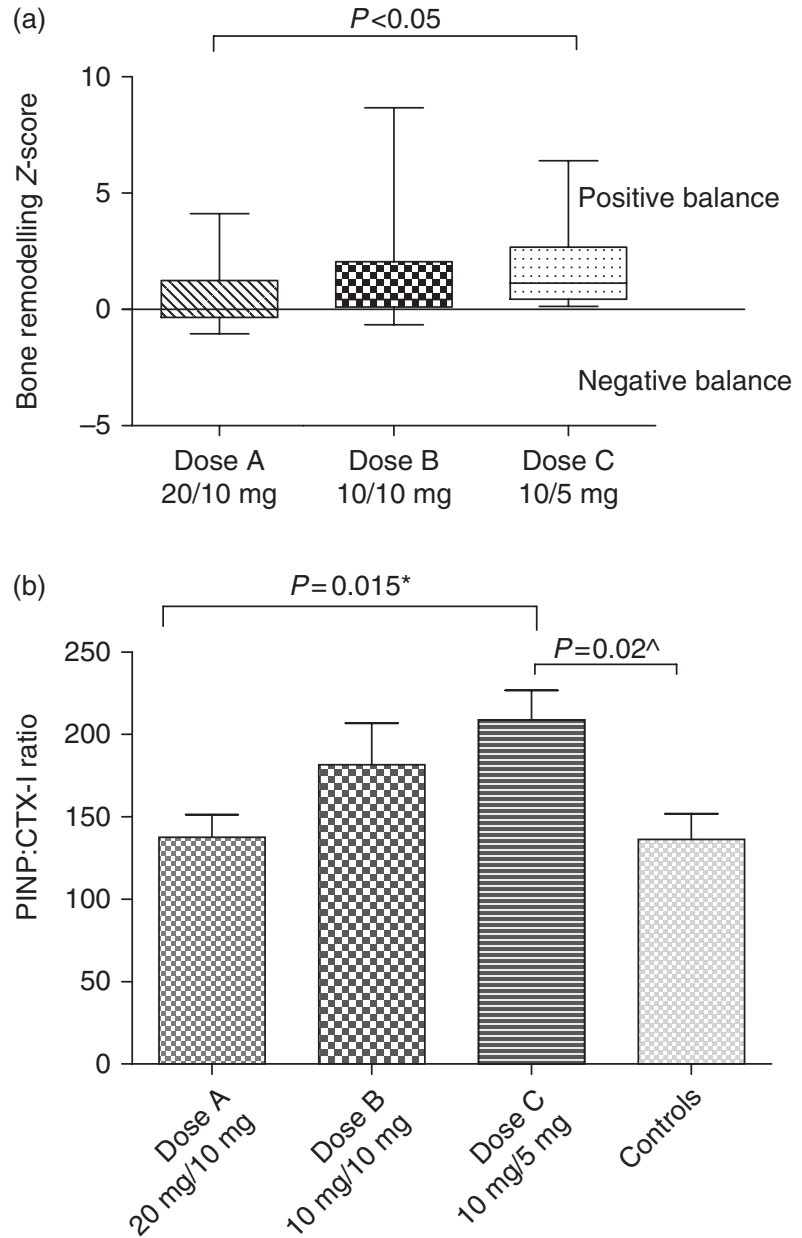

\section{Figure 3}

(a) Bone remodelling balance between dose regimens.

(b) PINP:CTX-I ratio between dose regimens and compared with controls. *Repeated-measures ANOVA between doses and $\wedge$ standard ANOVA compared with controls.

are still exposed to a wide range of GC replacement regimens; recent publications have reported the use of HC equivalent doses $>30 \mathrm{mg}$ daily in $67 \%$ (27) and $91 \%$ of the subjects (10) and $>20 \mathrm{mg}$ daily in $80 \%$ of the subjects in another study (28).

The measurement of bone turnover markers is associated with a number of pre-analytical factors that may affect it; therefore, to maintain the reliability of our results, we controlled for a number of these variables (29) by collecting the samples on each of the three occasions in a standardised setting under fasting conditions in the morning. Endocrine status affects bone turnover (29), and since our patients were on the full and stable modern pituitary replacement therapy, including for $\mathrm{GH}$, which was not altered during the study, we are confident that the observed alterations in bone turnover observed in our cohort are due to the effect of the alteration in HC dose during the study.

We demonstrated a $19-56 \%$ increase in the concentrations of OC(1-49), similar to the findings reported by Peacey et al. (13). Wichers also demonstrated an increase in the concentrations of OC(1-49) with a reduction in GC dose, significant for dose reduction from 30 to $20 \mathrm{mg}$ $(P<0.05)$ and from 30 to $15 \mathrm{mg}(P<0.01)$ after only 2 weeks on each treatment schedule (15) without alterations in the concentrations of any other bone turnover marker. In that double-blind, cross-over study in nine hypopituitary subjects, there was no control group and patients were GH deficient but not receiving GH replacement and there was no comment on the replacement of other pituitary hormones (15). Suliman et al. (30) examined the effect of three GC replacement schedules on bone turnover markers in nine subjects (one ACTH deficient): $\mathrm{HC} 10 \mathrm{mg}$ in the morning and $5 \mathrm{mg}$ in the evening; HC $10 \mathrm{mg}$ in the morning, $5 \mathrm{mg}$ at $1600 \mathrm{~h}$ and $5 \mathrm{mg}$ in the evening; and dexamethasone at $0.1 \mathrm{mg} / 15 \mathrm{~kg}$ body weight daily, by comparing the findings with those for the unmatched, younger, healthy controls. They demonstrated lower ionised calcium and higher 25OHD concentrations in subjects across all the replacement schedules compared with controls with no difference in PTH concentrations between subjects on different doses or between subjects and controls. Except for the reduced concentration of a resorption marker, urinary free deoxypyridinoline, in those receiving dexamethasone compared with those receiving $\mathrm{HC}$, there were no other differences in the markers of bone formation or resorption between schedules (30).

In the present study, the changes in bone formation correlated negatively with trough cortisol concentrations, rather than with peak levels, indicating that GC clearance may have a significant influence on bone health. Cortisol dynamics are likely to be as important as the overall prescribed dose, since studies on bone turnover and mineral density have demonstrated more pronounced adverse effects in patients on synthetic long-acting GCs (prednisolone and dexamethasone) than in those on $\mathrm{HC}$ or cortisone acetate $(9,12,30)$. Trials assessing metabolic outcomes using extended or modified release $\mathrm{HC}$ will be of interest (31).

Changes in the 'uncoupling index' or 'bone remodelling balance' have been shown to correlate with later changes in $\operatorname{BMD}(22,23)$, and a positive bone remodelling balance with associated increased bone turnover has been 
shown to be associated with increased bone formation and mineralisation. However, very high bone turnover is associated with an increased fraction of newly mineralised bone, which may be associated with suboptimal resistance (32). Observational studies $(33,34)$ have shown that there is an increase in bone resorption, without a concurrent increase in bone formation in men after the age of 60 years, and this imbalance is thought to be responsible for age-related bone loss in healthy men. We have demonstrated a more positive bone remodelling balance and an increased PINP:CTX-I ratio reflecting increased bone formation for low-dose HC regimen. The control subjects in the present study are likely to have quiescent bone milieu, and since hypopituitary patients are at a higher risk of bone loss than healthy men, increased bone formation suggests a favourable bone remodelling balance leading to net bone gain.

Whether the differences that we demonstrated in bone formation following dose reduction are likely to be maintained in the long term is unclear. Hermus et al. (35) determined, in nine premenopausal women with Cushing's disease, that surgical remission resulted in a $400 \%$ increase in the concentrations of OC(1-49). Increased bone formation was persistent at 2 years with an ongoing $100 \%$ increase in the concentrations of OC(1-49) from pre-operative levels. van Staa et al. (6) found that the relative rate of vertebral fracture decreased from 2.4 in the first year after discontinuation of GC therapy to 1.8 and subsequently returned to healthy control values thereafter. These findings indicate that the beneficial effects of GC dose reduction may be long standing.

There are a few limitations to the present study. It may not be possible to extrapolate the findings of the study to all severely ACTH-deficient subjects, because only male Caucasian subjects were studied. Our patient group had slightly reduced renal function than controls, and renal impairment is known to affect bone turnover and bone marker measurements, although this is usually only significant for GFR $<50 \mathrm{ml} / \mathrm{min}$ per $1.73 \mathrm{~m}^{2}$, in particular, for OC and CTX-I (29). The relatively reduced renal function in our cohort is unlikely to have significantly affected the results of the study as only one patient had a GFR $<60 \mathrm{ml} / \mathrm{min}$ per $1.73 \mathrm{~m}^{2}$ and there was no difference in renal function between patients under the three dose regimens, allowing accurate between-group comparison of $\mathrm{HC}$ effect on bone turnover markers, which is the primary aim of this study. Although two patients had controlled hypertension and were on angiotensin-converting enzyme inhibitor therapy, no patient in the whole group had evidence of overt secondary hyperparathyroidism and there was no correlation between renal function, PTH or 25OHD. We did demonstrate a weak negative correlation between 25OHD and bone turnover markers, except for TRACP5b, for the whole group. This finding may possibly reflect the effect of hypovitaminosis $\mathrm{D}$ on bone turnover markers, but it is unlikely to account for between-dose differences, as the correlation was lost when analysed for patients alone or between-dose regimens.

We had previously shown that in patients with severe ACTH deficiency a reduction in HC dose to $15 \mathrm{mg}$ daily leads to a greater number of physiological free cortisol profiles that mimic profiles of healthy matched controls without adversely affecting the quality of life (19). In this study, we have demonstrated a beneficial metabolic end organ effect of controlled HC dose reduction through increased bone formation and a positive bone remodelling balance. We recommend that physicians continue aiming to prescribe the lowest safely tolerated HC replacement regimen for subjects with severe ACTH deficiency. Thorough patient education regarding optimal management of $\mathrm{HC}$ replacement during intercurrent illness will better avoid hypoadrenal crises by appropriate acute GC management rather than through chronic overreplacement of $\mathrm{HC}$, thus helping to avoid the long-term adverse metabolic effects previously described with non-physiological GC replacement therapy.

\section{Supplementary data}

This is linked to the online version of the paper at http://dx.doi.org/10.1530/ EJE-13-0596.

\section{Declaration of interest}

The authors declare that there is no conflict of interest that could be perceived as prejudicing the impartiality of the research reported.

\section{Funding}

Dr L-A Behan was in receipt of an unrestricted educational grant from Pfizer Endocrine Care. Irish Medicines Board Clinical Trial Number СT900/459/1 and EudraCT Number - 2007-005018-37.

\footnotetext{
Author contribution statement

L-A Behan was responsible for study design and management, data collection and analysis, and manuscript preparation; G Kelleher carried out the biochemical analysis; M J Hannon contributed to data collection and manuscript preparation; J J Brady was involved in biochemical analysis and manuscript preparation; B Rogers was responsible for data collection and participant recruitment; W Tormey carried out the biochemical analysis; D Smith and C J Thompson were involved in manuscript preparation; and $\mathrm{M} J \mathrm{JcKenna}$ and $\mathrm{A}$ Agha are senior authors and were involved in the supervision of the study and manuscript preparation.
} 


\section{Acknowledgements}

The authors thank Prof. Dermot Kenny and the staff of the RCSI Clinical Research Centre, Dublin, Ireland, where the research was conducted, and Ms Patricia Barrett, Department of Chemical Pathology in Beaumont Hospital, Dublin, Ireland, for their assistance during the study.

\section{References}

1 Cushing $\mathrm{H}$. The basophil adenomas of the pituitary body and their clinical manifestations (pituitary basophilism) 1932. Obesity Research 19942 486-508. (doi:10.1002/j.1550-8528.1994.tb00097.x)

2 Plotz CM, Knowlton AI \& Ragan C. The natural history of Cushing's syndrome. American Journal of Medicine 195213 597-614. (doi:10.1016/ 0002-9343(52)90027-2)

3 Luengo M, Picado C, Del Rio L, Guaiiabens N, Montserrat JM \& Setoain J. Vertebral fractures in steroid dependent asthma and involutional osteoporosis: a comparative study. Thorax 199146 803-806. (doi:10.1136/thx.46.11.803)

4 McDougall R, Sibley J, Haga M \& Russell A. Outcome in patients with rheumatoid arthritis receiving prednisone compared to matched controls. Journal of Rheumatology 199421 1207-1213.

5 van Staa TP, Geusens P, Bijlsma JW, Leufkens HG \& Cooper C. Clinical assessment of the long-term risk of fracture in patients with rheumatoid arthritis. Arthritis \& Rheumatism 200654 3104-3112. (doi:10.1002/art. 22117)

6 van Staa TP, Leufkens HG, Abenhaim L, Zhang B \& Cooper C. Use of oral corticosteroids and risk of fractures. Journal of Bone and Mineral Research 200020 1487-1494. (doi:10.1002/art.22117)

7 Zelissen PM, Croughs RJ, van Rijk PP \& Raymakers JA. Effect of glucocorticoid replacement therapy on bone mineral density in patients with Addison's disease. Annals of Internal Medicine 1994120 207-221. (doi:10.7326/0003-4819-120-3-199402010-00005)

8 Braatvedt GD, Joyce M, Evans M, Clearwater J \& Reid IR. Bone mineral density in patients with treated Addison's disease. Osteoporosis International 199910 435-440. (doi:10.1007/s001980050251)

9 Løvås K, Gjesdal CG, Christensen M, Wolff AB, Almås B, Svartberg J, Fougner KJ, Syversen U, Bollerslev J, Falch JA et al. Glucocorticoid replacement therapy and pharmacogenetics in Addison's disease: effects on bone. European Journal of Endocrinology 2009160 993-1002. (doi:10.1530/EJE-08-0880)

10 Arlt W, Rosenthal C, Hahner S \& Allolio B. Quality of glucocorticoid replacement in adrenal insufficiency: clinical assessment vs. timed serum cortisol measurements. Clinical Endocrinology 200664 384-389. (doi:10.1111/j.1365-2265.2006.02473.x)

11 Jódar E, Valdepeñas MP, Martinez G, Jara A \& Hawkins F. Long-term follow-up of bone mineral density in Addison's disease. Clinical Endocrinology 200358 617-620. (doi:10.1046/j.1365-2265.2003.01761.x)

12 Koetz KR, Ventz M, Diederich S \& Quinkler M. Bone mineral density is not significantly reduced in adult patients on low-dose glucocorticoid replacement therapy. Journal of Clinical Endocrinology and Metabolism 201297 85-92. (doi:10.1210/jc.2011-2036)

13 Peacey SR, Guo CY, Robinson AM, Price A, Giles MA, Eastell R \& Weetman AP. Glucocorticoid replacement therapy: are patients over treated and does it matter? Clinical Endocrinology 199746 255-261. (doi:10.1046/j.1365-2265.1997.780907.x)

14 Chikada N, Imaki T, Hotta M, Sato K \& Takano K. An assessment of bone mineral density in patients with Addison's disease and isolated ACTH deficiency treated with glucocorticoid. Endocrine Journal 200451 355-360. (doi:10.1507/endocri.51.355)

15 Wichers M, Springer W, Bidlingmaier F \& Klingmüller D. How hydrocortisone substitution influences the quality of life and the bone metabolism of patients with secondary hypocortisolism. European Journal of Clinical Investigation 200030 55-57. (doi:10.1046/j.13652362.2000.0300s3055.x)
16 Verrotti A, Greco R, Morgese G \& Chiarelli F. Increased bone turnover in epileptic patients treated with carbamazepine. Annals of Neurology 200047 385-388. (doi:10.1002/1531-8249(200003)47:3<385::AIDANA18 $>3.0$. CO;2-W)

17 Szulc P \& Delmas PD. Biochemical markers of bone turnover: potential use in the investigation and management of postmenopausal osteoporosis. Osteoporosis International 200819 1683-1704. (doi:10.1007/ s00198-008-0660-9)

18 Perogamvros I, Aarons L, Miller AG, Trainer PJ \& Ray DW. Corticosteroidbinding globulin regulates cortisol pharmacokinetics. Clinical Endocrinology 201174 30-36. (doi:10.1111/j.1365-2265.2010.03897.x)

19 Behan LA, Rogers B, Hannon MJ, O'Kelly P, Tormey W, Smith D, Thompson CJ \& Agha A. Optimizing glucocorticoid replacement therapy in severely adrenocorticotropin-deficient hypopituitary male patients. Clinical Endocrinology 201175 505-513. (doi:10.1111/j.13652265.2011.04074.x)

20 Gertz BJ, Clemens JD, Holland SD, Yuan W \& Greenspan S. Application of a new serum assay for type I collagen cross-linked N-telopeptides: assessment of diurnal changes in bone turnover with and without alendronate treatment. Calcified Tissue International 199863 102-106. (doi:10.1007/s002239900497)

21 Qvist P, Christgau S, Pedersen BJ, Schlemmer A \& Christiansen C. Circadian variation in the serum concentration of C-terminal telopeptide of type I collagen (serum CTx): effects of gender, age, menopausal status, posture, daylight, serum cortisol, and fasting. Bone 200231 57-61. (doi:10.1016/S8756-3282(02)00791-3)

22 Eastell R, Robins SP, Colwell T, Assiri AM, Riggs BL \& Russell RG. Evaluation of bone turnover in type I osteoporosis using biochemical markers specific for both bone formation and bone resorption. Osteoporosis International 19933 255-260. (doi:10.1007/BF01623829)

23 Nanda KS, Ryan EJ, Murray BF, Brady JJ, McKenna MJ, Nolan N, O'Farrelly C \& Hegarty JE. Effect of chronic hepatitis C virus infection on bone disease in postmenopausal women. Clinical Gastroenterology and Hepatology 20097 894-899. (doi:10.1016/j.cgh.2009.01.011)

24 Josse AR, Atkinson SA, Tarnopolsky MA \& Phillips SM. Diets higher in dairy foods and dietary protein support bone health during diet- and exercise-induced weight loss in overweight and obese premenopausal women. Journal of Clinical Endocrinology and Metabolism 201297 251-260. (doi:10.1210/jc.2011-2165)

25 Anderson WJ, McFarlane LC \& Lipworth BJ. Prospective follow-up of novel markers of bone turnover in persistent asthmatics exposed to low and high doses of inhaled ciclesonide over 12 months. Journal of Clinical Endocrinology and Metabolism 201297 1929-1936. (doi:10.1210/ jc.2011-3410)

26 Lonergan R, Kinsella RK, Fitzpatrick P, Brady J, Murray B, Dunne C, Hagan R, Duggan M, Jordan S, McKenna M et al. Multiple sclerosis prevalence in Ireland: relationship to vitamin D status and HLA genotype. Journal of Neurology, Neurosurgery and Psychiatry 201182 317-322. (doi:10.1136/jnnp.2010.220988)

27 Sherlock M, Reulen RC, Aragon AA, Ayuk J, Clayton RN, Sheppard MC, Hawkins MM, Bates AS \& Stewart PM. ACTH deficiency, higher doses of hydrocortisone replacement, and radiotherapy are independent predictors of mortality in patients with acromegaly. Journal of Clinical Endocrinology and Metabolism 200994 4216-4223. (doi:10.1210/ jc.2009-1097)

28 Filipsson H, Monson JP, Koltowska-Haggstrom M, Mattsson A \& Johannsson G. The impact of glucocorticoid replacement regimens on metabolic outcome and co-morbidity in hypopituitary patients. Journal of Clinical Endocrinology and Metabolism 200691 3954-3961. (doi:10.1210/jc.2006-0524)

29 Seibel MJ. Biochemical markers of bone turnover: part I: biochemistry and variability. Clinical Biochemist Reviews 200526 97-122.

30 Suliman AM, Freaney R, Smith TP, McBrinn Y, Murray B \& McKenna TJ. The impact of different glucocorticoid replacement schedules on bone turnover and insulin sensitivity in patients with adrenal insufficiency. 
Clinical Endocrinology 200359 380-387. (doi:10.1046/j.1365-2265. 2003.01860.x)

31 Johannsson G, Nilsson AG, Bergthorsdottir R, Burman P, Dahlqvist P, Ekman B, Engström BE, Olsson T, Ragnarsson O, Ryberg $\mathrm{M}$ et al. Improved cortisol exposure-time profile and outcome in patients with adrenal insufficiency: a prospective randomized trial of a novel hydrocortisone dual-release formulation. Journal of Clinical Endocrinology and Metabolism 201297 473-481. (doi:10.1210/ jc.2011-1926)

32 Follet H, Boivin G, Rumelhart C \& Meunier PJ. The degree of mineralization is a determinant of bone strength: a study on human calcanei. Bone 200434 783-789. (doi:10.1016/j.bone.2003.12.012)
33 Szulc P \& Delmas PD. Biochemical markers of bone turnover in men. Calcified Tissue International 200169 229-234. (doi:10.1007/s00223001-1059-1)

34 Szulc P, Garnero P, Munoz F, Marchand F \& Delmas PD. Crosssectional evaluation of bone metabolism in men. Journal of Bone and Mineral Research 200116 1642-1650. (doi:10.1359/jbmr.2001. 16.9.1642)

35 Hermus AR, Smals AG, Swinkels LM, Huysmans DA, Pieters GF, Sweep CF, Corstens FH \& Kloppenborg PW. Bone mineral density and bone turnover before and after surgical cure of Cushing's syndrome. Journal of Clinical Endocrinology and Metabolism 199580 2859-2865. (doi:10.1210/jc.80.10.2859)

Received 21 July 2013

Revised version received 22 September 2013

Accepted 11 October 2013 